\title{
Coroas cerâmicas bi-layers: Uma revisão de literatura sobre tipos de cerâmicas, processamento cerâmico e tensão residual
}

\author{
Ceramic Crowns bi-layered: a review of \\ the literature on ceramics types, ceramic \\ processing and residual tension
}

\section{Alana Mirely Félix Moreira 1 (c) \\ Cicera Denise Pinheiro Bezerra Borges ${ }^{2}$ (1) Larissa Araújo Lopes Barreto ${ }^{3}$ (1) Manassés Tercio Vieira Grangeiro ${ }^{4}$ (1) Viviane Maria Gonçalves de Figueiredo ${ }^{5}$ (1)}

\author{
1,2Centro Universitário Leão Sampaio (Juazeiro do Norte). Ceará, Brasil. alanaigt@gmail.com, denisebezerra005@gmail.com \\ 3,4Universidade Estadual Paulista (São José dos Campos). São Paulo, Brasil. larissabarreto55@hotmail.com, terciomanasses@gmail.com \\ ${ }^{5}$ Autora para correspondência. Universidade Federal do Pernambuco (Recife).Pernambuco, Brasil. vivi_mfigueiredo@yahoo.com.br
}

\begin{abstract}
RESUMO | INTRODUÇÃo: O uso de materiais cerâmicos como estratégia restauradora enfrenta algumas problemáticas, como interferências entre a porcelana e zircônia, sendo a primeira utilizada como cerâmica de cobertura e a segunda como infraestrutura ou coping. OBJETIVO: revisar a literatura sobre coroas cerâmicas bi-layer, através dos tipos de cerâmicas, processamento cerâmico e tensão residual. METODOLOGIA: As bases de dados para esta revisão foram Bireme, Pubmed, Scielo e Bibliotecas Virtuais. As palavras-chaves foram buscadas no Mesh, quanto aos critérios de inclusão são Estudos, Laboratoriais e Clínicos, Revisão Sistemática e Metanálise, artigos científicos e literatura específica sobre o tema, literatura em inglês e português. Já os critérios de exclusão foram carta ao editor, caso clínico e artigo de opinião, literaturas que não abordasse o tema em estudo e artigos que abordem outras cerâmicas odontológicas. A literatura e os artigos foram selecionados por meio de resumos e abstracts. CONSIDERAÇÕES FINAIS: Tensão residual em coroas cerâmicas bi-layers, com infra-estrutura em zircônia e cobertura em porcelana, ocorrem pela ação de diversos fatores como coeficiente de expansão térmica, velocidade de resfriamento, processamento e espessura da cerâmica de cobertura.
\end{abstract}

PALAVRAS-CHAVE: Zircônia. Porcelana. Resistência mecânica. Resistência adesiva.

\begin{abstract}
INTRODUCTION: The usage of ceramic materials as a restoration strategy faces some problems, such as interferences between porcelain and zirconia, being the first one used as ceramic cover and the second one as infrastructure or coping. OBJECTIVE: review the literature on bi-layer ceramic crowns, through the types of ceramics, ceramic processing and residual stress. METHODOLOGY: Databases for this review were Bireme, Pubmed, Scielo and Virtual Libraries. Keywords were searched on Mesh. Inclusion criteria were Studies, Laboratorial and Clinical, Systematic Review and Meta-Analysis, papers and specific literature regarding the theme, in both English and Portuguese. Exclusion criteria were letter to the editor, clinical case and opinion piece, literatures that don't face the theme and papers that analyze other odontological ceramics. Literature and papers were selected through summaries and abstracts. FINAL CONSIDERATIONS: Residual tension in bi-layers ceramics crowns with zirconia infrastructure and porcelain cover occur due to a lot of factors such as coefficient of thermal expansion, cooling speed, processing, and thickness of ceramic cover.
\end{abstract}

KEYWORDS: Zirconia. Porcelain. Mechanical resistance. Adhesive resistance. 


\section{Introdução}

As cerâmicas odontológicas são materiais bastante utilizados na reabilitação oral de pacientes que apresentam necessidades restauradoras e/ou substituição de um elemento dental perdido. Tais cerâmicas restabelecem estética, pela alta capacidade de mimetizar os tecidos dentais, longevidade, estabilidade de cor e biocompatibilidade. A procura por restaurações estéticas tem aumentado o uso dessas cerâmicas dentais, esse material tem excelência em reproduzir a anatomia dentária, antes apresentava baixa resistência mecânica, que limitava sua indicação. Atualmente existem cerâmicas com propriedades mecânicas mais favoráveis que permitem sua indicação em regiões de maior carga oclusa鹏.

A odontologia moderna vem sofrendo mudanças graduais que acompanham paralelamente os padrões da sociedade, no que se refere à função e estética. Desde a criação das cerâmicas até a atualidade esses materiais sofreram diversas modificações na tentativa de melhorar seu desempenho mecânico e com isso surgiram novas formas de processamento. Os avanços tecnológicos permitiram a ampliação da indicação dos sistemas cerâmicos, a produção manual foi sendo substituída pelo uso da informática a favor da odontologia.

No entanto o uso de materiais cerâmicos como estratégia restauradora enfrenta algumas problemáticas, como por exemplo a incompatibilidade (tensão residual) entre a porcelana e zircônia, sendo a primeira utilizada como cerâmica de cobertura e a segunda como infra-estrutura ou copingf. Vários estudos na literatura têm mostrado uma elevada incidência de fratura da porcelana sobre a zircônia, decorrente desta incompatibilidade térmica. Tal condição pode ser desencadeada devido a tensão residual constituídas em coroas bi-layers com coping de zircônia com aplicação de porcelana, durante o resfriamento do processamento cerâmico a alta temperatura国.

Com base na problemática abordada e devido à escassez de estudos sobre os fatores que levam a falha de coroas bi-layer, o presente trabalho permite compreender os fatores que interferem no processamento de coroas metal free com infra-estrutura em zircônia e cobertura em porcelana. Objetivou-se revisar a literatura sobre coroas cerâmicas bi-layer, através dos tipos de cerâmicas, processamento cerâmico e tensão residual.

\section{Metodologia}

As palavras-chaves foram buscadas no Mesh, sendo assim selecionadas as seguintes: Zircônia (Zirconia); Porcelana (Porcelain); Resistência Mecânica (Mecanical Strength), Resistência Adesiva (Adhesion Strenght).

Os critérios de inclusão e exclusão dos artigos científicos para esta revisão são apresentados no Quadro 1.

Quadro 1. Critérios de inclusão e exclusão utilizados para a revisão de literatura

\begin{tabular}{|l|l|}
\hline \multicolumn{1}{|c|}{ Critérios de Inclusão } & \multicolumn{1}{c|}{ Critérios de Exclusão } \\
\hline \begin{tabular}{l|l|} 
Estudos Laboratoriais e Clínicos Revisão \\
Sistemática \\
Metanálise
\end{tabular} & $\begin{array}{l}\text { Carta ao editor } \\
\text { Caso Clínico } \\
\text { Artigo de Opinião } \\
\text { Revisão de Literatura }\end{array}$ \\
\cline { 1 - 2 } $\begin{array}{l}\text { Artigos científicos e literatura específica sobre o } \\
\text { tema }\end{array}$ & $\begin{array}{l}\text { Literatura que não aborda como variável o processamento } \\
\text { cerâmico }\end{array}$ \\
\hline \multicolumn{1}{|c|}{ Literatura em inglês e português } & $\begin{array}{l}\text { Literatura que aborde outras cerâmicas odontológicas que } \\
\text { não são o foco do estudo }\end{array}$ \\
\hline
\end{tabular}


A seleção da análise do conteúdo foi realizada com base na leitura de resumos ou abstracts, definindo assim a literatura que será incluída e excluída nesta revisão. Bem como, artigos duplicados em base de dados diferentes será contabilizado apenas uma vez. Não houve uma determinação de ano de publicação na seleção da literatura, por ser uma temática ainda pouco difundida.

\section{Revisão de literatura}

\section{Cerâmicas Odontológicas}

As cerâmicas odontológicas vêm tornando-se a principal opção de tratamento para reabilitação de uma estrutura dental perdida. A escolha desse material tem algumas razões: baixa condutibilidade térmica e elétrica, resistência à compressão, tais quais são quimicamente inertes e produz uma excelente mimetização dos dentes naturais, atendendo as requisições estéticas e funcionais].

Na época atual há uma ampla diversidade de tipos de cerâmicas acessíveis para as mais diversas indicações, embora não tenha nenhum tipo de cerâmica que possa ser indicado para todas as circunstâncias clínicas, que possam surgir. Incentivadas por uma busca pela estética do mercado, brevemente as empresas passaram a fabricar cerâmicas para infra-estrutura que podiam ser aplicadas em situações clínicas que há uma maior exigência de carga oclusal|?

A história da cerâmica na Odontologia mostra como ocorreu seu progresso e como isso caminhava paralelo à necessidade da existência de um material que fosse estético, mas ao mesmo tempo contivesse boas propriedades mecânicas, foi preciso que houvesse mudanças na sua composição e forma de processamento. Apesar das cerâmicas possuírem boas características, ocorria falhas no que diz respeito à fragilidade e a baixa resistência mecânica quando submetida às tensões de tração. Tais tensões eram acumuladas nas extremidades, nos ângulos e nas fendas da restauração. Outro fator de escolha desse material é a sua translucidez, quanto mais translúcida a cerâmica for, provavelmente ela será indicada em casos onde há grande requisição estética. Porém, ao aumentar a translucidez, sua resistência a fratura diminui, por se tratarem de grandezas inversamente proporcionais?.
Devido às falhas clínicas, principalmente fraturas catastróficas que ocorriam com as cerâmicas, houve a necessidade de melhorias nesse material. Isso foi possível graças ao aperfeiçoamento dos métodos de confecção e pela adição de maiores frações e fases cristalinas .

As cerâmicas podem ser constituídas por vidros, porcelanas, cerâmicas vítreas e estruturas cristalinas, quanto a sua degradação de superfície podem se classificar em: Cerâmicas ácido-sensíveis e cerâmicas ácido-resistentes押.

As cerâmicas ácido-sensíveis (feldspáticas, dissilicato de lítio e as leucíticas) são passíveis de condicionamento com ácido fluorídrico a $10 \%$, que favorece a união aos cimentos resinosos, apresentando uma resistência adesiva satisfatória, sendo indicadas para facetas, lentes de contato, coroas anteriores, inlay e onlay. Por ter maior conteúdo vítreo, apresenta meIhores características estéticasion.

Já as cerâmicas ácido-resistentes, Alumina e Zircônia, apresentam melhores propriedades mecânicas por ter alto teor cristalino, assim necessitam de um tratamento de superfície mais específico antes da cimentação adesiva. Tais característica se deve ao fato de que o ácido fluorídrico a 10\% não é capaz de degradar sua superfície, por conter pouco ou nenhuma quantidade de sílica, assim são indicadas para infra-estrutura, coroas unitárias de dentes posteriores[0].

As cerâmicas podem ter maior quantidade de matriz vítrea ou cristalina, quanto mais matriz cristalina, maior a resistência das restaurações, com esse raciocínio as cerâmicas reforçadas por cristais possuem melhores características mecânicas quando comparadas aquelas não reforçadas. Os cristais que são normalmente adicionados são a alumina, leucita, dissilicato de lítio, spinel e zircônia, que aumentam a resistência reduzindo a formação de trincas e fraturas quando sujeitas a forças desfavoráveis, por outro lado, quando se ganha em resistência se perde em estética devido as cerâmicas ficarem mais opacas朋.

A expansão térmica significa o aumento dimensional do material, enquanto o coeficiente de expansão térmica linear (CETL) é a alteração existente no comprimento por unidade de comprimento de determinado material a cada $1^{\circ} \mathrm{C}$ que sua temperatura se eleva. Em próteses cerâmicas e metalocerâmicas o material de 
cobertura deve ter o CETL semelhante ao da infraestrutura, para que se contraiam e se expandam de maneira igual, quando houver variação de temperatura. Os diversos tipos de cerâmicas odontológicas variam entre suas propriedades térmicas, químicas, físicas e mecânicas e suas vantagens estão relacionadas ao desempenho de suas propriedades mecânicas, estéticas e pela sua biocompatibilidade ${ }^{\text {I }}$.

As cerâmicas ácido-sensíveis se classificam em Feldspática e Dissilicato de Lítio, enquanto as cerâmicas ácido-resistentes se classificam em alumina e zircônia].

\section{Cerâmica Feldspática}

As porcelanas variam muito quanto a sua composição e dependendo dos elementos que estão em maior número as indicações mudam, na odontologia os elementos mais presentes são feldspato e quartzo, que conferem melhores propriedades ópticas favorecendo a estética 14 . O feldspato geralmente não é encontrado na sua forma pura, sendo que para uso na odontologia, é preferível o que contém alto conteúdo de Potássio, pois nessa forma quando submetido a temperaturas elevadas tem grande resistência ao escoamento. Quando fundido, algumas partículas de álcalis começam a se unir a alumina e a sílica, e acabam formando silicato de sódio e silicato de potássio. A preferência do feldspato para a odontologia acontece principalmente por se tratar de um material de fácil obtenção, e a variação da pigmentação do pó, que favorece totalmente a estética. $O$ feldspato em altas temperaturas ainda forma a leucita (silicato-potássio-alumínio), que apresenta alto CETL e são compatíveis com o das ligas metálicas, sendo vantajoso para próteses metalocerâmicas ${ }^{9}$. As cerâmicas feldspáticas foram desenvolvidas na Inglaterra, sendo as primeiras a serem confeccionadas em alta fusão, mas com o tempo foi se verificando que ela apresentava baixa resistência mecânica e isso limitou suas indicações, passando a ser indicada basicamente para coroas unitárias anteriores?

Para melhoria de suas características, ela que é baseada na sílica, foi adaptada com reforço de leucita, aumentando seu coeficiente de expansão térmico e aumentando suas indicações para coroas unitárias, inlay e onlay, em restaurações confeccionadas totalmente em cerâmica . Conforme houve progresso nos materiais odontológicos, em 1990 foram desenvolvidas as cerâmicas reforçadas por leucita, com a adição de 40 a $55 \%$ de cristais de leucita à porcelana convencional, possuindo melhor resistência a flexão que a das cerâmicas feldspáticas estando por volta de 90 a $180 \mathrm{MPa}$ apresentam boas propriedades óticas e podem ser indicadas para confecção de inlays, onlays e facetas laminadas e coroas unitárias anteriores e nos pré-molares, podendo também ser usada para confecção de coroas metalocerâmicas por possuir coeficiente de expansão térmica similar ao de ligas áuricas神四.

As cerâmicas acima citadas foram as primeiras a serem empregadas para confecção de próteses e restaurações, apresentavam ótima qualidade estética devido à translucidez, coeficiente de expansão térmica semelhante aos dos dentes, não possuíam potencial corrosivo e foram utilizadas por um longo período, porém sua indicação era limitada devido à baixa resistência mecânica mediante forças de tração (60Mpa), a desvantagem de uma porcelana convencional é que as partículas são sinterizadas simultaneamente, resultando em microporosidades dentro do próprio material. Como consequência, essas desuniformidades podem iniciar a propagação de fissuras, levando à falência precoce das restaurações, sendo indicadas para coroas unitárias anteriores, situação que apresenta menor quantidade de forças oclusais].

\section{Cerâmica Aluminizada}

A resistência relativamente baixa da porcelana fez com que se desenvolvesse as cerâmicas aluminizadas por McLean e Hughes no ano de 1965 aumentando a fase cristalina pela adição de óxidos de alumina, essas cerâmicas apresentaram a resistência à flexão duplicada (130Mpa) quando comparada às feldspáticas. A alumina reduz a concentração das forças na parte interna da peça, que geralmente ocorre no resfriamento, e também possuem temperatura de queima maior, conferindo maior resistência mecânica e diminuindo a formação de trincas, porém aumentou significativamente a opacidade da cerâmica, tornando-a menos estética que a feldspática,e esse material também não é indicado para regiões de grande esforço mastigatório间. 
Com o objetivo de tentar minimizar os problemas relacionados à resistência houve a introdução no mercado de um novo sistema cerâmico infiltrado por vidro, sistema In-Ceram (Vita Zahnfabrik, Sackingen, Alemanha), que ocorreu após a proposta das cerâmicas alumizadas. A resistência flexural aumentou em até 4 vezes em comparação às outras cerâmicas, podendo chegar a $650 \mathrm{Mpa}$, possui duas fases: fase alumina (óxido de alumino) e fase vítrea (óxido de lantânio), sendo, portanto, cerâmicas de alta resistência. Nesse sistema há a produção de uma alumina porosa que depois é infiltrada por vidro, mas esses materiais apresentam pouca ou nenhuma fase vítrea, essa infiltração extingue a porosidade residual e atribui à infraestrutura grande resistência mecânica. O sistema In-Ceram apresenta até 3 variações: InCeram Alumina, spinel (MgAl2O4) - In-Ceram Spinel e zircônia (Al2 O3 ZrO2) - In-Ceram Zircônia役国

No ano de 1995 a Vita ${ }^{\circledR}$ lançou no mercado o sistema In Ceram Alumina ${ }^{\circledR}$ que apresenta uma composição de alumina variando entre $70 \%$ a $85 \%$ com resistência à flexão variando de 450-600 Mpa, é indicado para coroas unitárias posteriores e anteriores e PPR de até três elementos na região anterior (de central a canino). O In -Ceram Zircônia compreende cerca de 33 a $35 \%$ de óxido de zircônio que confere melhores propriedades mecânicas a esse material apresentando uma resistência à flexão de até $800 \mathrm{Mpa}$, só que apresenta grande opacidade limitando sua capacidade estética, sendo indicado apenas para regiões posteriores, ele pode ser utilizado para confeccionar prótese parcial fixa de até três elementos ou coroas únicas. O InCeramspinel é constituída por alumina e por espinélio de magnésio, este constitui a maior parte da fase cristalina, esse tipo de cerâmica apresenta uma resistência à flexão inferior ao do sistema In Ceram Alumina, por volta de 280 a $380 \mathrm{Mpa}$, porém possui propriedades estéticas melhores quando comparada à alumina e à zircônia, graças às propriedades isotrópicas do aluminato de magnésio e da parte vítrea, que melhoram a translucidez da peça, a sua confec-

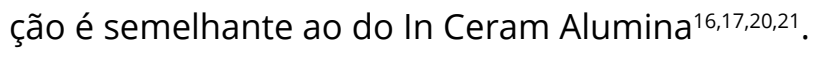

Cerâmica Dissilicato de Lítio Outro tipo de cerâmica que foi desenvolvido foram as reforçadas por dissilicato de lítio que apresentam a adição desses cristais em até $65 \%$ na sua composição, o que aumentou consideravelmente as propriedades mecânicas desse material elevando a resistência a flexão para até 300 a 400 Mpa. Têm indicação para inlays, onlays, laminados, coroas unitárias e próteses parciais fixas de três elementos até a região de $2^{\circ}$ pré-molar, apresentam boa estética e uma resistência favorávell.

\section{Cerâmica Zircônia}

A zircônia é um tipo de cerâmica odontológica que se apresenta em três formas: monoclínica, cúbica e tetragonal e é muito indicada para uso restaurador pela boa estabilidade química e dimensional, resistência mecânica e por sua dureza semelhante ao aço inoxidável. Tal cerâmica apresenta maior tenacidade à fratura, dureza elevada e mais resistente ao desgastę2. A zircônia parcialmente estabilizada por óxido de ítrio (YTZP) têm sido bastante utilizadas nos dias atuais na confecção de infraestruturas de coroas e próteses parciais fixas

Apesar desta cerâmica apresentar vantagens estéticas em relação à infraestrutura de metal, por ser extremamente branca precisa de uma cerâmica de coberta, que seja uma cerâmica vítrea que permita passagem de luz equivalente ao esmalte dentário, a porcelana. A reabilitação usando Y-TZP pode variar o número de peças utilizadas, dependendo do número de dentes perdidos e de acordo com cada fabricante, possui resistência a flexão variando de 900 a 1200 Mpa, o que possibilita reabilitar mais de três elementos em região posterior. 


\begin{tabular}{|c|c|c|c|}
\hline Classificação & $\begin{array}{l}\text { Tipos de } \\
\text { Cerâmica }\end{array}$ & Características & Indicação \\
\hline \multirow{2}{*}{ 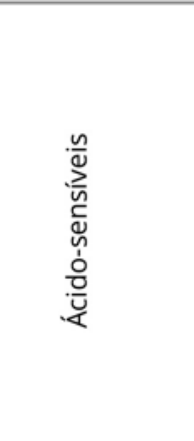 } & $\begin{array}{l}\text { Feldspática } \\
\text { (Porcelana) }\end{array}$ & $\begin{array}{l}\text { Qualidade estética. } \\
\text { Baixa resistência. }\end{array}$ & $\begin{array}{l}\text {-Coroas unitárias anteriores para } \\
\text { recobrimento de infraestrutura metálica } \\
\text { ou cerâmica em situações de pequeno } \\
\text { stress oclusal. }\end{array}$ \\
\hline & $\begin{array}{l}\text { Dissilicato } \\
\text { de Lítio }\end{array}$ & $\begin{array}{l}\text { Adesividade ao substrato } \\
\text { dental. } \\
\text { Ótima resistência flexural. } \\
\text { Melhor resistência quando } \\
\text { comparada a feldspática. }\end{array}$ & $\begin{array}{l}\text {-Inlays e onlays. } \\
\text {-Coroas unitárias. } \\
\text {-Facetas laminadas. } \\
\text {-Próteses fixas de três elementos até o } \\
\text { segundo pré-molar. }\end{array}$ \\
\hline \multirow{2}{*}{ 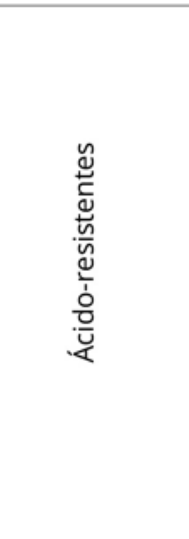 } & Alumina & $\begin{array}{l}\text { 2x mais resistentes se } \\
\text { comparadas à feldspática. } \\
\text { Menos translucidez devido aos } \\
\text { cristais dealumina. } \\
\text { Pouco indicada para região } \\
\text { posterior. }\end{array}$ & $\begin{array}{l}\text {-Núcleos cerâmicos. } \\
\text {-Coping/infraestrutura de coroas } \\
\text { unitárias e próteses parciais fixas }\end{array}$ \\
\hline & Zircônia & $\begin{array}{l}\text { Sem união química para } \\
\text { técnicas adesivas. } \\
\text { Elevada Resistência a Fratura. } \\
\text { Opaco. } \\
\text { Boa resistência mecânica. } \\
\text { Processado pelo Cad-Cam. Alto } \\
\text { Módulo de Elasticidade. }\end{array}$ & $\begin{array}{l}\text {-Casos que exigem preparos mais } \\
\text { amplos. }\end{array}$ \\
\hline
\end{tabular}

Fonte: Os autores (2020).

\section{Processamento Cerâmico}

As formas de processamento também são uma das maneiras de classificação das cerâmicas, pois cada uma apresenta um método específico de trabalho com o uso do material correto para cada caso, algumas das maneiras de confecção são: estratificação (condensação, por meio da técnica do pó e líquido); injeção/prensagem; ou (CAD-CAM) fresagem/usinagemes.

Durante a técnica do pó e líquido as restaurações de porcelanas são construídas pelo método tradicional da junção de pó e líquido fornecido pelo fabricante, sendo o pó constituído de porcelana e o líquido modelador que é a mistura de água destilada com modificadores. Condensados em uma placa de vidro, uma pasta é formada e aplicada em camadas por um pincel, com o cuidado de continuar condensando a cada camada pela remoção da água em excesso. Após a construção da peça de porcelana, esta segue para um forno específico de porcelanas odontológicas onde acontece a sinterização ou queima para que ocorra a união das partículas do pó e aumento da densidade da massa para redução da porosidade. Esse processo deve garantir a redução da quantidade de água sem que a peça sofra deformação. Por fim, a peça é ajustada no modelo ou paciente e pode passar por um glazeamento, que é a aplicação de uma fina camada de porcelana com ou sem pigmentos com a finalidade de que as trincas e falhas superficiais sejam seladas?.

O sistema cerâmico prensado/injetado é fundamentado na técnica da cera perdida, nela há um padrão de cera ou resina acrílica que tem o formato da restauração que é incluído em sistema refratário e suprimido em forno com altas temperaturas, assim no final é obtido espaço suficiente para a cerâmica, esta será posicionada na forma de pastilhas e posta sobre alta temperatura em um forno específico para ser injetada no molde, assim a cerâmica ocupa o espaço que foi obtido anteriormente formando a restauração indireta. Essa técnica utilizando alta pressão facilitou a resolução dos problemas de contração que ocorriam na sinterização das cerâmicas feldspáticas, assim a variação de dimensão só ocorre durante 
resfriamento, essa técnica aumentou a resistência das cerâmicas utilizadas. O dissilicato de lítio e as cerâmicas reforçadas por leucita são os principais tipos

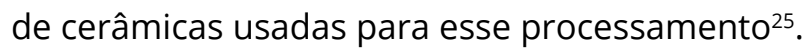

Desde a década de 1970 o sistema CAD/CAM vem sendo investigado para uso na Odontologia. Inicialmente acreditava-se que a tecnologia teria mais fácil manuseio na odontologia que nas indústrias, mas tudo um dou quando foi avaliado o custo, tempo de operação, a precisão para projetar e processar as restaurações e a morfologia necessária dos dentes pilaresę.

CAD/CAM (Computer Aided Design/ Computer Aided Manufacturing) é o nome dado a uma técnica onde acontece basicamente a digitalização em um modelo de gesso ou da própria boca do paciente e o arquivo é enviado à uma máquina e fresados por meio de uma combinação de códigos e brocas diamantadas que cortam um bloco cerâmico. O CEREC foi o primeiro sistema a ser comercializado e pode ser encontrado em duas modalidades: Chairside para clínica e inLab específico para laboratório. No sistema PROCERA, o arquivo digitalizado é enviado ao laboratório de processamento na Suécia ou nos E.U.A. onde são feitas réplicas mais alargadas do modelo de gesso para compensar a contração da cerâmica durante a sinterização. No LAVA a digitalização é feita através de um laser e a estrutura é desenhada com aumento de $20 \%$ do seu tamanho para dessa forma compensara contração da cerâmica. Por fim o EVEREST onde a digitalização é feita por uma câmera CCD e a imagem 3D criada através de 15 sequências de projeção e a fresagem do bloco cerâmico realizado por movimentos de corte de cinco eixos?t.

Ainda nos avanços computacionais da Odontologia, a moldagem digital está apresentando vantagens significativas para a odontologia pois substitui a moldagem convencional feita com a confecção de modelos de gesso por um método bem mais confortável para o paciente, reduzindo o tempo de trabaIho, ocupando menos espaço físico com o acúmulo de modelos de gesso, diminuindo a quantidade de erros por formação de bolhas, ruptura do material, distorção, além de ser mais econômico. Estão disponíveis atualmente no mercado duas técnicas para moldagem digital: Os sistemas de moldagem tridimensional e os sistemas CAD/CAM. Após escaneados, os modelos digitais podem ser arquivados e armazenados no próprio disco rígido?.
A utilização dos scanners intra orais representam um grande avanço na Odontologia e atualmente podem ser adicionadas informações obtidas por tomografia computadorizada cone beam (CBCT), que é o mais promissor método de diagnóstico por imagem para odontologia, essa associação permite melhor cópia dos tecidos orais do paciente, possibilitando menor margem de erro e produção de restaurações mais adaptadas 29. As inovações na odontologia têm aumentando exponencialmente nas últimas décadas devido à incorporação de tecnologias digitais nas mais diversas especializações. A era digital chegou para revolucionar a Odontologia, a introdução da tecnologia CAD/CAM possibilitou a transformação de trabalhos manuais em um sistema automatizado, não no sentido de uma produção em série, mas sim num aperfeiçoamento na produção das restaurações pelo uso de desenhos e processamentos orientados por computador. Atualmente, as cerâmicas, de acordo com os procedimentos laboratoriais de fabricação, são divididas em cinco categorias: cerâmicas convencionais, fundidas, prensadas, infiltradas e computadorizadas $\beta$.

Os procedimentos computadorizados usam o sistema CAD/CAM que vem sendo um método inovador desde a sua criação, há cerca de 40 anos, trazendo entre suas vantagens a diminuição do tempo clínico e o aperfeiçoamento do trabalho laboratorial. Esse sistema foi desenvolvido para a criação de subestruturas de próteses cerâmicas utilizando óxido de alumínio altamente purificado e densamente sintetizado formado por mais de $99,5 \%$ de alumina. O desenho da estrutura protética é feito em um computador seguido de sua confecção por uma máquina de fresagem. O uso desse sistema na Odontologia tem sido viável principalmente na confecção de facetas, coroas e pontes. De acordo com a disponibilidade de ceder os arquivos CAD: sistemas CAD-CAM abertos ou CAD-CAM fechados. A vantagem de um sistema aberto é a possibilidade de poder escolher o sistema CAM mais adequado aos propósitos, pois é possível transmitir o arquivo CAD para outro computador. Os sistemas CADCAM fechados oferecem todo o sistema de produção?. 
Atualmente há uma grande variedade de sistemas CAD CAM disponíveis para uso na rotina clínica do cirurgião dentista, esses sistemas apresentam vantagens que tornam a sua utilização proveitosa para muitos procedimentos odontológicos. Diversos estudos clínicos são realizados para comprovar a eficácia dessa tecnologia, que vem evoluindo gradativamente com o tempo, mostrando que esses sistemas podem sempre melhorar as limitações fazendo uso das inovações tecnológicas 2 .

A técnica RapidLayer Technology é um processamento recente para a cerâmica de cobertura, consiste em uma técnica simples para confecção de coroas e pontes de cerâmica pura por meio de um bloco de cerâmica de alta qualidade e sendo usinado por CAD/CAM, podendo construir estruturas de pontes de até 4 elementos na região posterior. O sistema usa a tecnologia do inovador programa de computador Sirona in Lab 3D Software $\geq$ V3.80, e o resultado do trabalho é obtido em 3 etapas. Primeiro é feito um escaneamento dos elementos dentários e a imagem é enviada para computador onde as espessuras ideais dos materiais já são fornecidos, em seguida a subestrutura é fresada através de um bloco de dióxido de zircônio de VITA InCeram YZ seguido de sua sinterização em um forno de alta temperatura, depois a cerâmica de cobertura é fresada e é utilizado blocos VITABLOCS TriLuxe Forte, que são confeccionados a partir de uma cerâmica de longa comprovação clínica, os VITABLOCS, que possuem ótimas características estéticas. A infraestrutura e a cerâmica de cobertura obtidas se encaixam perfeitamente. Por último se utiliza cimentos resinosos convencionais para cimentação da cobertura sobre a in-

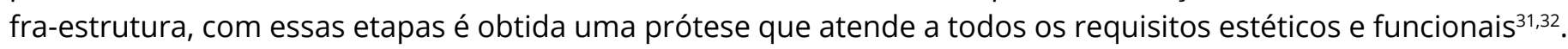

Na técnica do RapidLayer, com utilização dos VITABLOCS TriLuxe Forte, que são camadas finas de ceramic feldspática para recobrimento das estruturas de zircônia, o fabricante garante a ausência de desarmonias térmicas e de falhas na cerâmica. Ao optar por essa técnica, coroas com $2 \mathrm{~mm}$ de espessura apresentam desempenho melhor quanto a fratura, que coroas com $1 \mathrm{~mm}$ (Lima, 2014). A cobertura sendo fresada em CAD-CAM elimina o trabalho manual e otimiza a confecção laboratorial demonstrada na Figura 1.

Figura 1. Técnica do Rapid-layer, infraestrutura em zircônia e cerâmica de cobertura, tratamento de superfície na porção externa da infraestrutura e interna da cobertura e cimentação resinosa sobre a zircônia

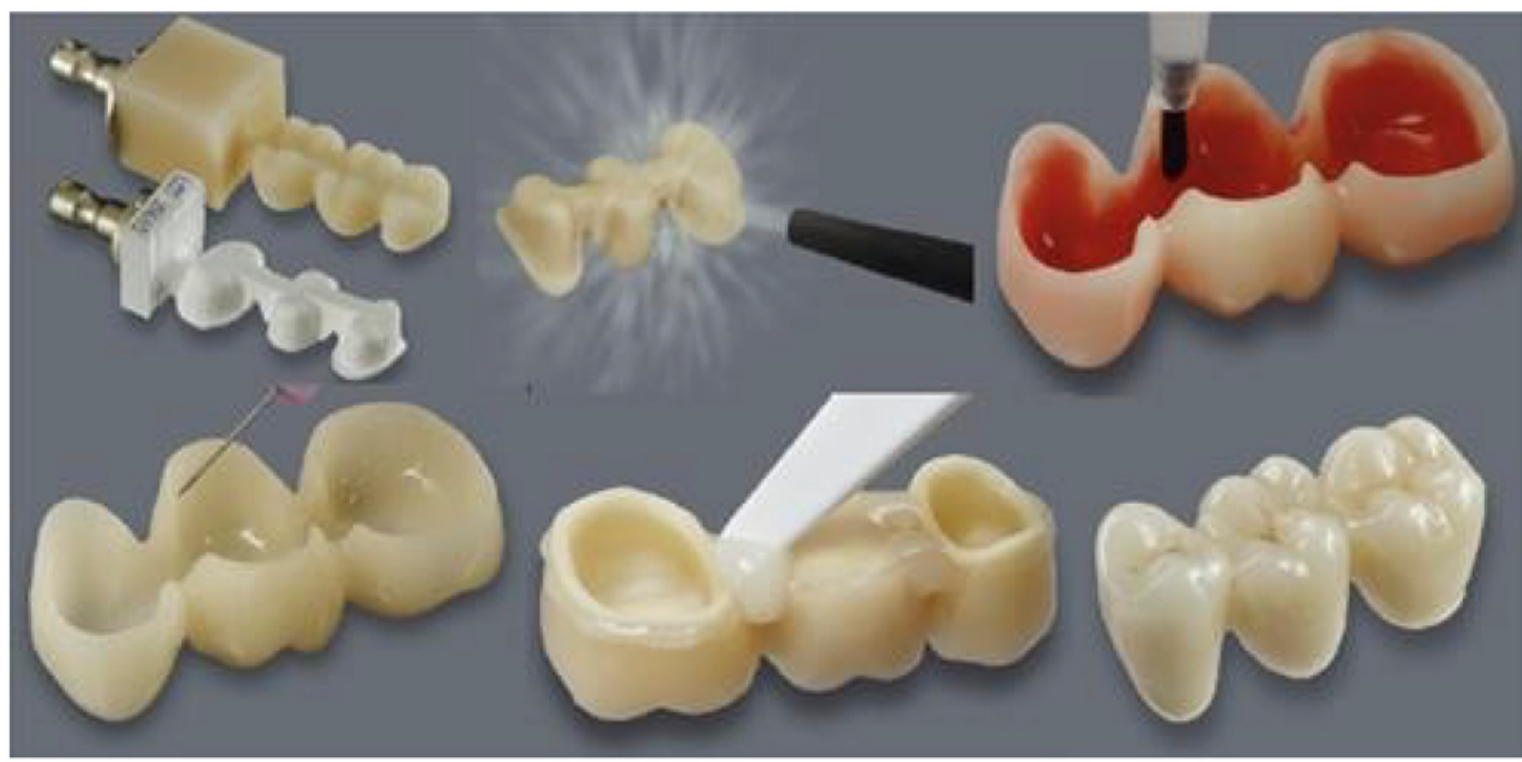

Fonte: https://www.vita-zahnfabrik.com. 
Estudos clínicos que relatam o resultado de restaurações à base de zircônia indicam que a resistência a fratura da cerâmica ácido-resistente é suficiente para suportar forças mastigatórias. Contudo, tem sido relatada uma incidência significativa de fratura coesiva da porcelana de recobrimento sobre a infraestrutura em Y-TZP. Há associações positivas entre a ocorrência de fissuras e as três variáveis (materiais, espessura e taxa de resfriamento, coeficiente de expansão térmica) 3 .

Já no estudoß34 revelaram a presença de um estresse de tração radial na porcelana/cerâmica de cobertura, o que contribui para as grandes fraturas clínicas observadas nessas próteses livres de metal com infra-estrutura em zircônia.

As principais complicações dos sistemas cerâmicos à base de zircônia são o lascamento da porcelana, que se verificou estar diretamente associada ao desenvolvimento de tensões térmicas residuais na camada de porcelanaß5.

As tensões residuais se desenvolvem na fase de aquecimento, devido à sequência de efeitos dos gradientes térmicos na qual a fase líquida da cobertura se torna sólida e ocorre um desajuste nas propriedades do coeficiente de expansão térmica entre zircônia e a porcelana. Essa solidificação ocorre de fora para dentro, portanto, não é uniforme e isso causa uma compressão dentro da peça. Tais tensões diminuem à medida que se aprofundam, sendo mais compressivas na superfícię. A Figura 2 representa a tensão residual entre porcelana e zircônia, e a seguir serão apresentados os estudos revisados.

Figura 2. Esquema representativo da tensão residual em uma coroa cerâmica bi-layer, com infra- estrutura em zircônia e cobertura em porcelana

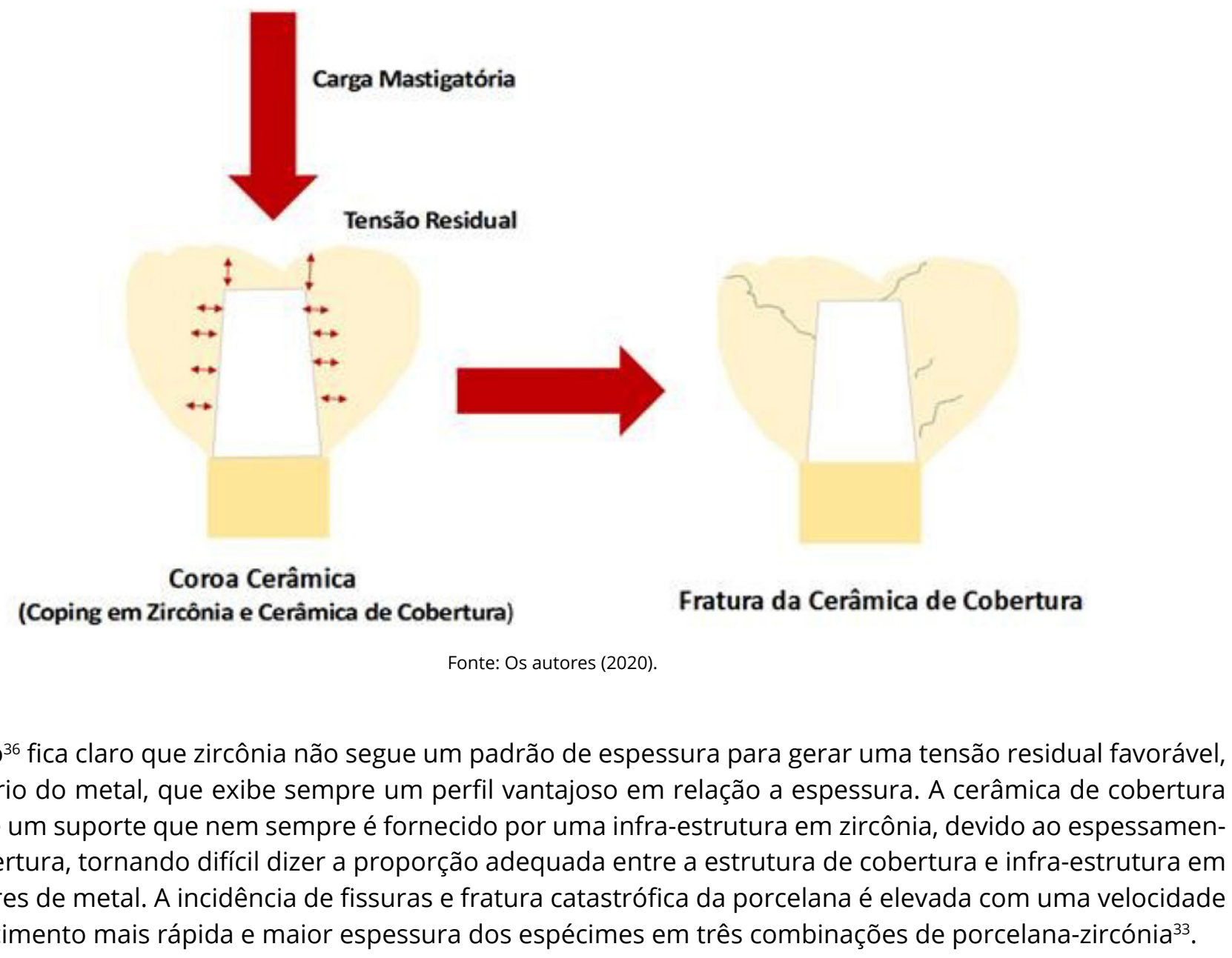

No estudo 36 fica claro que zircônia não segue um padrão de espessura para gerar uma tensão residual favorável, ao contrário do metal, que exibe sempre um perfil vantajoso em relação a espessura. A cerâmica de cobertura precisa de um suporte que nem sempre é fornecido por uma infra-estrutura em zircônia, devido ao espessamento da cobertura, tornando difícil dizer a proporção adequada entre a estrutura de cobertura e infra-estrutura em coroas livres de metal. A incidência de fissuras e fratura catastrófica da porcelana é elevada com uma velocidade de arrefecimento mais rápida e maior espessura dos espécimes em três combinações de porcelana-zircónia ${ }^{33}$. 
Através de um estudo31 in vitro analisou fatores que poderiam influenciar a tensão residual, como diferentes técnicas de aplicação, protocolos de resfriamento e a espessura da cerâmica de cobertura e observou que a técnica prensada com taxa de resfriamento lenta foi o método que obteve melhores resultados em relação à fratura da peça. As peças confeccionadas pelo sistema CAD/CAM usando o Rapid Layer apresentou resistência a fratura menor que as técnicas prensada e estratificada.

Já no estudoßj observou a resistência a flexão, tenacidade a fratura e estresse residual dentre dois processamentos distintos de porcelana sobre uma infraestrutura de zircônia, de acordo com os resultados a porcelana prensada apresentou superioridades mecânicas em relação a porcelana aplicada pela técnica do pó e líquido.

Enquanto que o estudoß to extremamente lento elevou a tensão residual em relação ao resfriamento lento recomendado pelo fabricante, portanto um procedimento demorado que não favorecerá a mecânica da fratura.

Estudo in silico 3D observou o desempenho de diferentes materiais restauradores para restabelecer um primeiro molar inferior, com aplicação de carga axial e valor semelhante a carga mastigatória em populações ocidentais. As coroas apresentavam-se em ouro, metalocerâmica, alumina, zircônia e um compósito. As coroas totalmente cerâmicas apresentaram a menor distribuição de tensão para o preparo dental em relação as demais coroas. Observou-se que estes materiais têm um efeito sobre o padrão de distribuição de tensão dentro do complexo restauração-dente quando submetidos a diferentes condições de carga. Coroas em zircônia exibiram o pico mais alto em valores de tensão de von Mises, induzidos dentro do material restaurador. Isto indica que estas coroas sofreram valores de tensão máximos antes de os distribuir para a estrutura do dente subjacente].

Por meio de Elementos Finitos estudou-se as tensões estáticas sobre coroas, com coping em zircônia + compósito e coping em zircônia + porcelana, os achados não mostraram diferença entre os valores de Tensões de Von Mises. Porém, tal estudo apresentou limitações por ser uma avaliação estática, estudos com carregamento a longo prazo devem ser buscado, a fim de avaliar outras variáveis entre tais estratégias restauradoras

No estudoß日 in Silico em bi-layers cerâmicas observou-se as tensões residuais entre zircônia/porcelana, e alumina /porcelana, os últimos apresentaram a presença de menor estresse residual que os primeiros. Na busca de solucionar tal situação, o uso de um conjunto tri-layer com uma camada intermediária para amortecer tais tensões, favorece a distribuição de tensão residual, a qual afeta a resistência a fratura de uma coroa totalmente cerâmica. O risco de falha para sistemas de restauração dentária totalmente cerâmicos pode ser significativamente reduzido usando sistemas tri-layer (gradiente intercalar), assim devese buscar estudos que simulem as condições orais para testar tal condição.

O uso de uma camada graduada entre porcelana e zircônia pode ter um impacto significativo no desempenho clínico das próteses livre de metal, promovendo a redução da incidência de fratura da zircônia e da delaminação da porcelanatô.

As restaurações de zircônia apresentaram valores de tensão térmica maior do que as de alumina. As tensões térmicas são significativamente reduzidas pela presença de uma camada intermediária homogênea, reduzindo a chance de falhas catastróficas 9 .

Outros fatores que podem interferir a incompatibilidade entre estas duas cerâmicas odontológicas é que a zircônia após a sinterização pode ainda apresentar porcentagens de fase monoclínica, o que favorece a tensão residual com a porcelana $4^{\text {11 }}$.

$\mathrm{Na}$ tentativa de reduzir tais condições de fratura o tratamento de superfície externa da infra-estrutura (jateamento com óxido de alumínio) em zircônia parece favorece a adesão da porcelanat2. Enquanto o estudd ${ }^{3}$ mostra que a vitrificação pode ser considerada uma opção para aumentar a resistência de união entre zircônia-porcelana, alcançando os maiores valores de resistência de união.

O Quadro 3 representa a descrição dos estudos revisados acima. 


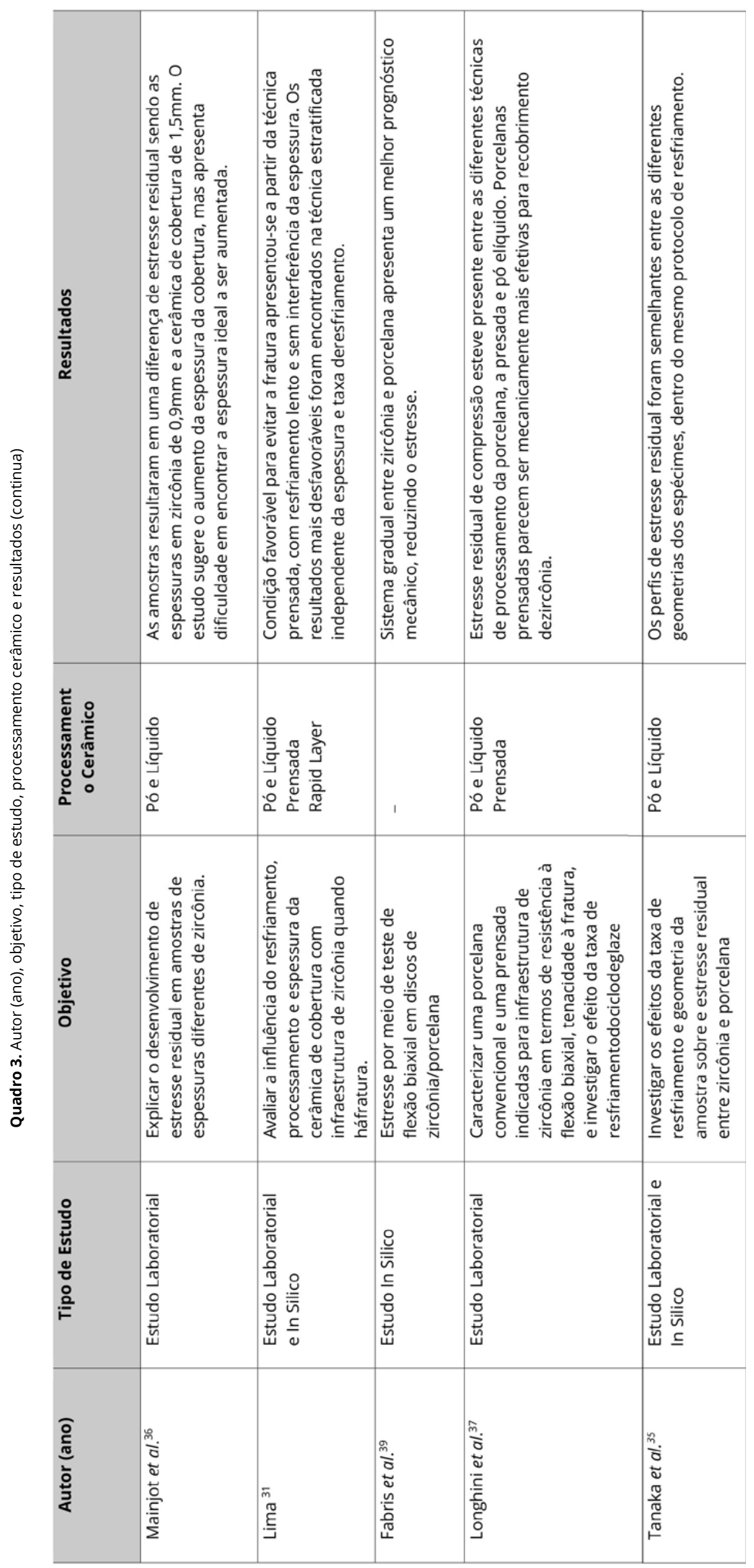

J. Dent. Public. Health, Salvador, 2020 Junho;11(1):52-66

Doi: 10.17267/2596-3368dentistry.v11v1.2880 | ISSN: 2596-3368 


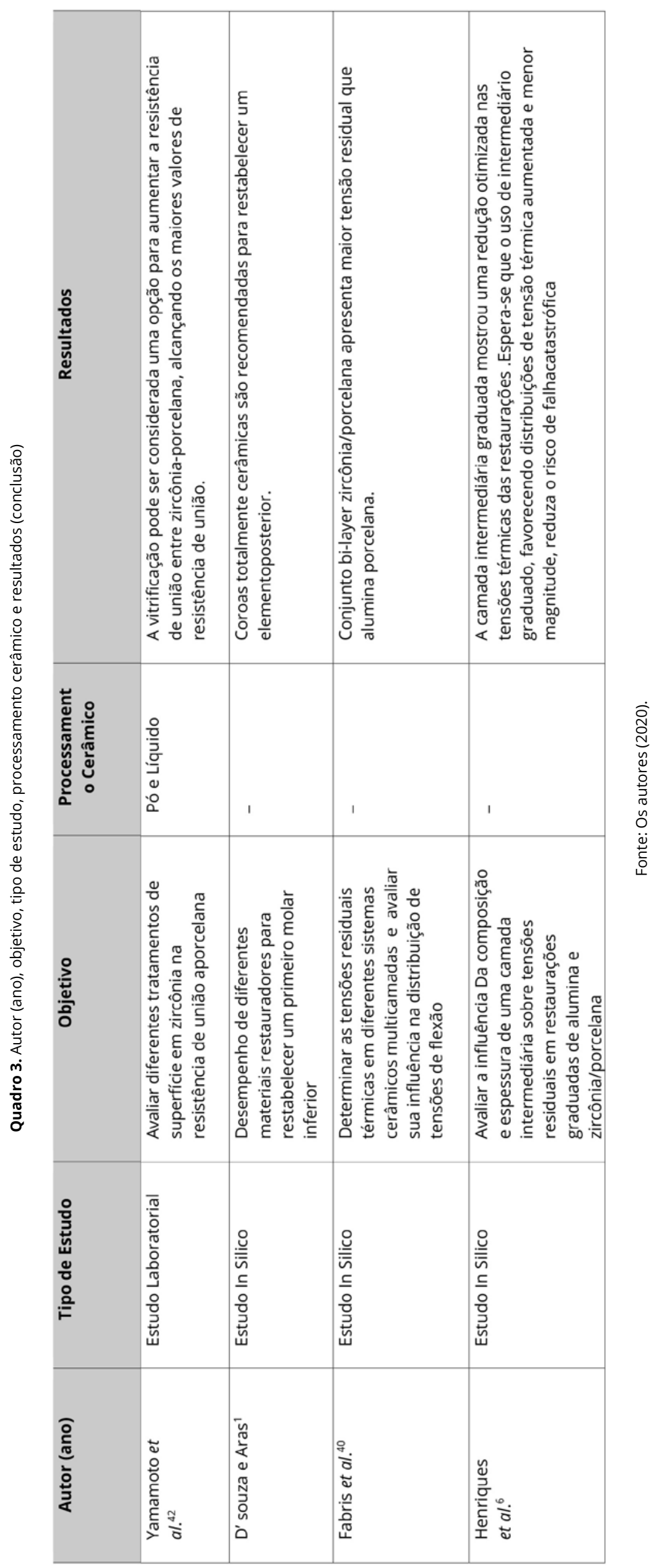

J. Dent. Public. Health, Salvador, 2020 Junho;11(1):52-66

Doi: $10.17267 / 2596-3368$ dentistry.v11v1.2880 | ISSN: 2596-3368 
A elevada frequência de fraturas catastróficas da cerâmica de cobertura sobre uma infraestrutura em zircônia, tem sido achado frequentes nas clínicas odontológicas, porém devido à recente consolidação das restaurações livres de metal, tal temática tem sido pesquisada nos últimos anos, na busca de encontrar soluções para esta problemática. Os estudos revisados são todos laboratoriais ou in Silico, sendo este fator uma limitação para compreender o que ocorre intra-oral, se fazendo necessários estudos clínicos].

Com base na literatura, os fatores citados anteriormente podem atuar para favorecer a tensão residual na porcelana e assim gerar a fratura, mas as evidências científicas apontam que uma camada como gradiente entre porcelana e zircônia possa ser a solução destes problemas. Porém, são indícios que necessitam de novas evidências, bem como o uso da técnica do rapid layer, a qual praticamente observou-se apenas uma literatura sobre o assunto, que deve ser mais bem investigada.

A ação de tratamentos de superfície entre ambas cerâmicas parece melhorar a união entre as mesmas, porém a tensão residual não é questão de adesão e sim ação de incompatibilidade entre propriedades cerâmicas, assim até quando este tratamento superficial é benéfico, também deve ser investigado, pois o jateamento induz a mudança de fase da zircônia.

\section{Considerações finais}

Tensão residual em coroas cerâmicas bi-layer, com infra-estrutura em zircônia e cobertura em porcelana, ocorrem pela ação de diversos fatores como coeficiente de expansão térmica, velocidade de resfriamento, processamento e espessura da cerâmica de cobertura. Deve-se avaliar se a reabilitação com esse sistema livre de metal deveria ter tanta aplicação no dia-a-dia clinico levando em consideração que ele tem alto custo para o paciente e não possui a longevidade esperada. O cirurgião-dentista deve estar atualizado sobre essas condições e possíveis inovações, principalmente para escolher melhor o processamento e o resfriamento que gerem menor tensão residual por fim alcançar o sucesso clínico.

\section{Contribuição dos autores}

Moreira MFM, Borges CDPB, Barreto LAL responsabilizaramse pela escrita do artigo. Grangeiro MTV e Figueiredo VMG responsabilizaram pela correção do artigo.

\section{Conflitos de interesses}

Nenhum conflito financeiro, legal ou político envolvendo terceiros (governo, empresas e fundações privadas, etc.) foi declarado para nenhum aspecto do trabalho submetido (incluindo, mas não se limitando a subvenções e financiamentos, participação em conselho consultivo, desenho de estudo, preparação de manuscrito, análise estatística, etc.).

\section{Referências}

1. D' Sousa KM, Aras MA. Three-dimensional finite element analysis of the stress distribution pattern in a mandibular first molar tooth restored with five different restorative materials. J Indian Prosthodont Soc. 2017;7(1):53-60. doi: 10.4103/09724052.197938

2. Martins LM, Lorenzoni FC, Farias BC, Lopes LDS, Bonafante G, Rubo JH. Comportamento biomecânico das cerâmicas odontológicas: revisão. Cerâmica. 2010;56(338):148-155. doi: 10.1590/S0366-69132010000200009

3. Gomes EA, Assunção WG, Rocha EP, Santos PH. Cerâmicas odontológicas: o estado atual. Cerâmica. 2008;54(331):319-325. doi: 10.1590/S0366-69132008000300008

4. Rueda AO, Seuba J, Anglada JSM, Jiménez-Piqué E. Tomography of indentation cracks in feldspathic dental porcelain on zirconia. Dental Mater. 2013;29(3):348-56. doi: 10.1016/j. dental.2013.01.001

5. Örtorp A, Kihl ML, Carlsson GE. A 3-year retrospective and clinical follow-up study of zirconia single crowns performed in a private practice. J Dent. 2009;37(9):731-6. doi: 10.1016/j. jdent.2009.06.002

6. Henriques B, Fabris D, Souza JCM, Silva FS, MesquitaGuimarães J, Zhang Y et al. Influence of interlayer design on residual thermal stresses in trilayered and graded all-ceramic restorations. Materials Science and Engineering: C. 2017;71:10371045. doi: $\underline{10.1016 / j . m s e c .2016 .11 .087}$

7. Chain MC, Arcari GM, Lopes GC. Restauraçöes cerâmicas estéticas e próteses livres de metal: as novas alternativas possibilitadas pelas novas porcelanas. RGO. 2000;48(2):67-70.

8. Garcia LFR, Consani S, Cruz PC, Souza FCPP. Análise crítica do histórico e desenvolvimento das cerâmicas odontológicas. RGO. 2011;59:67-73. 
9. Amoroso AP, Ferreira MB, Torcato LB, Pellizzer EP, Mazaro JVQ, Gennari Filho H. Cerâmicas odontológicas: propriedades, indicações e considerações clínicas. Revista odontológica de Araçatuba. 2012;33(2):19-25.

10. Bottino MA, Faria R, Valandro LF. Percepção: estética em próteses livres de metal em dentes naturais e implantes. Brasil: Artes Médicas; 2009.

11. Hipolito AC, Ferreira MB, Barão VA, Faco EFS, Delben JA, Santos $\mathrm{PH}$ et al. Cimentação em próteses cerâmicas livres de metal: o estado atual e perspectivas futuras. Rev Odontol UNESP. 2012;41:117.

12. Anusavice KJ, Shen C, Rawl HR. Philips Materiais Dentários. 12.ed. Elsevier: Brasil; 2013.

13. Mackert Jr JR, Russell CM. Leucite crystallization during processing of a heat-pressed dental ceramic. Int J Prosthodont. 1996;9(3):261-5.

14. Kwlly JR, Benetti P. Ceramic materials in dentistry: historic al evolution and current practice. Aust Dent J. 2011;56(Suppl 1):8496. doi: $10.1111 / j .1834-7819.2010 .01299 . x$

15. Cattell MJ, Chadwick TC, Knowles JC, Clarke RL, Lynch E. Flexural strength optimisation of a leucite reinforced glass ceramic. Dent Mater. 2001;17(1):21-33. doi: 10.1016/s01095641(00)00046-4

16. Bohjalian A, Froner EE, Zanetti AL, Santos VMA. Resistência à fratura de sistemas cerâmicos Empress I, II e In-Ceram: estudo sobre fatores envolvidos nos testes. RGO. 2006;54(2):185-190.

17. Giordano RA, Pelletier L, Campbell S, Pober R. Flexural strength of an infused ceramic, glass ceramic, and feldspathic porcelain. J Prosthet Dent. 1995;73(5):411-8. doi: 10.1016/S00223913(05)80067-8

18. Bona AD, Kelly JR. The clinical success of all-ceramic restorations. J AM Dent Assoc. 2008;139:S8-S13. doi: 10.14219/ jada.archive.2008.0361

19. Font AF, Ruiz FS, Ruíz MG, Rueda CL, González AM. Choice of ceramic for use in treatments with porcelain laminate veneers. Choice of ceramic for use intreatments with porcelain laminate veneers. Med Oral Patol Oral Cir Bucal 2006;11(3):297-302.

20. Chong KH, Chai J, Takahashi Y, Wozniak W. Flexural strength of In-Ceram alumina and In-Ceram zircônia core materials. Int J Prosthodont. 2002;15(2):183-8.

21. Bona AD, Shen C, Anusavice KJ. Work of adhesion of resin on treated lithia disilicate-based ceramic. Dent Mater. 2004;20(4):33844. doi: 10.1016/S0109-5641(03)00126-X
22. Francisco MG. Propriedades mecânicas de cerâmicas de zircônia tetragonal policristalina contendo ítrio (Y-TZP) após aplicação de cerâmica. [dissertação]. São Paulo: Universidade de Taubaté; 2009.

23. Guazzato M, Quach L, Albakry M, Swain MV. Influence of surface and heat treatmentson the flexural strength of Y-TZP dental ceramic. J Dent. 2005;33(1):9-18. doi: 10.1016/j. jdent.2004.07.001

24. Conceição EN. Restaurações estéticas: compósitos, cerâmicas e implantes. Porto Alegre: Artes médicas; 2005, P. 308.

25. Craig RG, Powers JM. Materiais dentários restauradores. 11.ed. São Paulo: Santos; 2004.

26. Miyazaki T, Hotta Y, Kunii J, Kuriyama S, Tamaki Y. A review of dental CAD/CAM: current status and future perspectives from 20 years of experience. Dental Materials Journal. 2009;28(1):44-56. doi: $10.4012 / \mathrm{dmj} .28 .44$

27. Correia ARM, Fernandes JCAS, Cardoso JAP, Silva CFCL. CAD-CAM: a informática a serviço da prótese fixa. Revista de Odontologia da UNESP. 2006;35(2):183-89.

28. Polido WD. Moldagens digitais e manuseio de modelos digitais: o futuro da Odontologia. Dental Press J Orthod. 2010;15(5):18-22.

29. Karl M, Graef F, Wichmann M, Krafft T. Passivity of fit of CAD/ CAM and copy-milled frameworks, veneered frameworks, and anatomically contoured, zirconia ceramic, implant-supported fixed prostheses. J Prosthet Dent. 2012;107(4):232-8. doi: 10.1016/S0022-3913(12)60067-5

30. Rosenblum MA, Schulman A. A review of all-ceramic restorations. J Am Dent Assoc. 1997;128(3):297-307. doi: 10.14219/jada.archive.1997.0193

31. Lima JMC. Efeito de diferentes espessuras, técnicas de aplicação e protocolos de resfriamento na resistência à fratura da cerâmica de cobertura aplicada sobre infraestrutura de y-tzp. [tese]. São Paulo: Universidade Estadual Paulista Julio de Mesquita Filho; 2014.

32. VITA. VITA Rapid Layer Technology. Disponível em: https://www.vita-zahnfabrik.com/en/VITA-Rapid-LayerTechnology-41926,27568.html

33. Guazzato M, Walton TR, Franklin W, Davis G, Bohl C, Klineberg I. Influenc of hickness and ling rate on development of spontaneous cracks in porcelain/zirconia structures. Aust Dent J. 2010;55(3):306-10. doi: 10.1111/j.1834-7819.2010.01239.x

34. Baldassarri M, Stappert CFJ, Wolff MS, Thompson VP, Zhang Y. Residual stresses in porcelain-veneered zirconia prostheses. Dent Mater. 2012;28(8):873-9. doi: 10.1016/j.dental.2012.04.019 
35. Tanaka CB, Harisha $\mathrm{H}$, Baldassarri M, Wolff MS, Tong $\mathrm{H}$, Meira JBC et al. Experimental and finite element study of residual thermal stresses in veneered Y-TZP structures. Ceram Int. 2016;42(7):9214-9221. doi: 10.1016/j.ceramint.2016.03.018

36. Mainjot AK, Schajer GS, Vanheusden AJ, Sadoun MJ. Influence of veneer thickness on residual stress profile in veneering ceramic: measurement by hole-drilling. Dental materials. 2012;28(2):160-67. doi: 10.1016/j.dental.2011.11.008

37. Longhini D, Rocha COM, Medeiros IS, Fonseca RG, Adabo GL. Effect of glaze cooling rate on mechanical properties of conventional and pressed porcelain on zirconia. Braz Dent J. 2016;27(5):524-531. doi: 10.1590/0103-6440201600709

38. Alsadon O, Patrick D, Johnson A, Pollington S, Wood D. Fracture resistance of zirconia-composite veneered crowns in comparison with zirconia-porcelain crowns. Dent Mater J. 2017;36(3):289-295. doi: 10.4012/dmj.2016-298

39. Fabris D, Souza JCM, Silva FS, Fredel M, Mesquita-Guimarães J, Zhang $Y$ et al. Thermal residual stresses in bilayered, trilayered and graded dental ceramics. Ceramics international. 2017;43(4):3670-3678. doi: 10.1016/j.ceramint.2016.11.209

40. Fabris D, Souza JC, Silva FS, Fredel M, Mesquita-

Guimarães J, Zhang Y et al. Thermal residual stresses in bilayered, trilayered and graded dental ceramics. Ceramics international. 2017;43(4):3670-3678. doi: 10.1016/j. ceramint.2016.11.209

41. Ryan DPO, Fais LMG, Antonio SG, Hatanaka GR, Candido LM, Pinelli LAP. Y-TZP zirconia regeneration firing: Microstructural and crystallographic changes after grinding. Dent Mater J. 2017;36(4):447-453. doi: 10.4012/dmj.2016-124

42. Mosharraf R, Rismanchian M, Savabi O, Ashtiani AH. Influence of surface modification techniques on shear bond strength between different zirconia cores and veneering ceramics. J Adv Prosthodont. 2011;3(4):221-228. doi: 10.4047/jap.2011.3.4.221

43. Yamamoto LT, Rodrigues VA, Dornelles LS, Bottino MA, Valandro LF, Melo RMD. Low-fusing porcelain glaze application on 3Y-TZP surfaces can enhance zirconia-porcelain adhesion. Braz Dent J. 2016;27(5):543-547. doi: 10.1590/0103-6440201601014 